\title{
Healing effect of andiroba-based emulsion in cutaneous wound healing via modulation of inflammation and transforming growth factor beta $\mathbf{3}^{1}$
}

Chang Yung Chia', Andréia Dantas Medeiros", André de Menezes Silva Corraes", José Eduardo Ferreira Manso"', César Silveira Claudio da Silva"', Christina Maeda Takiya'v, Ricardo Luís Vanz

'MD, Postgraduate Program in Surgical Science, Department of Surgery, School of Medicine, and Immunopathology Laboratory, Instituto de Biofísica Carlos Chagas Filho, Universidade Federal do Rio de Janeiro (UFRJ), Brazil. Conception and design of the study, analysis and interpretation of data, statistics analysis, technical procedures, manuscript writing, critical revision.

"PhD, Instituto de Biofísica Carlos Chagas Filho, UFRJ, Rio de Janeiro-RJ, Brazil. Technical procedures, manuscript writing, critical revision, final approval.

I'PhD, Postgraduate Program in Surgical Science, Department of Surgery, School of Medicine, UFRJ, Rio de Janeiro-RJ, Brazil. Design of the study, analysis and interpretation of data, final approval.

IVPhD, Postgraduate Program in Surgical Science, Department of Surgery, School of Medicine, and Immunopathology Laboratory, Instituto de Biofísica Carlos Chagas Filho, UFRJ, Rio de Janeiro-RJ, Brazil. Design of the study, analysis and interpretation of data, immunohistochemical examinations, statistics analysis, manuscript writing, critical revision, final approval.

${ }^{\vee} \mathrm{MD}$, Postgraduate Program in Surgical Science, Department of Surgery, School of Medicine, UFRJ, Rio de Janeiro-RJ, Brazil. Manuscript writing, critical revision, final approval.

\section{Abstract}

Purpose: To evaluate the effects and mechanisms of andiroba-based emulsion (ABE) topical treatment on full-thickness cutaneous wounds in rats.

Methods: The wounds were harvested on days 3, 7, 15, and 20 post-surgery. Wound contraction rate, quantitative immunohistochemistry [macrophages, myofibroblasts, capillaries, collagens (col) I and III, transforming growth factor $\beta 3 \beta$ (TGF 33 )], and tensile strength were assessed.

Results: Treated wounds were smaller, contracted earlier and had increased angiogenesis, fewer CD68+ and M2 macrophages on days 7 and 15, but higher on day 20. Myofibroblasts appeared on days 3 to 7 in untreated wounds and on days 7 to 15 in treated wounds. TGF 3 levels were higher in the treated wounds, less dense collagen fibers, lower col I/III ratios and a higher tensile strength.

Conclusion: These results demonstrate the important anti-inflammatory role of treatment and the associated modulation of macrophages, myofibroblasts, and TGF $\beta 3$ levels. Collagen fibers in the treated wounds were more organized and less dense, similar to unwounded skin, which likely contributed to the higher tensile strength.

Key words: Cicatrix. Myofibroblasts. Plants, Medicinal. Wound Healing. Macrophages. Rats. 


\section{Introduction}

In humans, skin full-thickness injuries evolve to healing with scar formation. Despite the high capacity of skin to regenerate, humans have lost this capacity. Consequently, healed wounds may generate scars, which is a major clinical problem, resulting in loss of function, adverse cosmesis, and hindrance of growth in children. Scars also have a substantial impact on a patient's quality of life and have been associated with anxiety, social avoidance, and depression, in addition to representing a significant medical burden. Cutaneous scars commonly occur after surgical operations, trauma, burns and infections.

Regeneration or healing without scars, as similarly seen in the fetus ${ }^{1}$, constitutes the goal of medical wound care. Wound healing is a complex, dynamic process that demands cell-cell and cell-matrix interactions that are regulated by several signaling pathways. Moreover, the sequential progression of the healing process is impaired in chronic wounds. After injury, blood fills the wound, and fibrin together with plasma fibronectin constitutes the provisional matrix enabling the host-recruited blood marrow cells. Neutrophils infiltrate the wound during the first 24 hours, followed by macrophages, which populate the wound in large numbers, peaking at two days post-injury. Macrophages eat dead neutrophils, cell debris, and foreign organisms, and they secrete several growth factors. These molecules, together with chemokines and cytokines produced in situ, provide an adequate microenvironment for the growth of new capillaries and fibroblasts as well as generate a provisional extracellular matrix (ECM). Altogether, these constituents form the granulation tissue. At this stage, from the edges of the wound, epithelial cells begin to proliferate to re-epithelize wounds. Macrophages constitute the predominant inflammatory cells after 72 hours and seem to have a crucial role in the response to injury due to their plasticity. These cells have key roles in coordinating later events, favoring the appearance of myofibroblasts and ECM synthesis, mainly the collagen network, which ultimately forms the bulk of the mature scar. This abnormal ECM primarily differs from that of unwounded skin and mature scars in its collagen content, containing increased levels of immature type III collagen (relative to the mature type I collagen that predominates in normal skin and mature scars. The ability of myofibroblasts to adhere to each other allows them to contract, which makes these cells the most appropriate to provide conditions for wound contraction. Subsequently, the newly deposited ECM is remodeled. However, the synthesis of ECM is not completely stopped but is considerably reduced and modified. Collagen type III, the major component of the granulation tissue, is gradually replaced by collagen type I, which is the main structural component of the dermis. In the resolution phase of healing, the cell number is dramatically reduced by apoptosis of both vascular cells and myofibroblasts. The wound is now composed of thick collagen bundles distributed in a disorganized pattern, and the scar has only $70-80 \%$ of its original tensile strength, which is insufficient for the development of new melanocytes and the skin adnexae, such as sweat glands and hair follicles.

Despite great knowledge on the physiopathology of cutaneous healing, it is still a challenge since no mechanisticbased therapies currently exist to prevent or improve scarring, except for the use of recombinant TGF $33^{2}$. Current therapies, such as pressure garments, silicone dressings, and hydrocortisone injections, are empirical, unpredictable and largely ineffective ${ }^{3}$.

Alternative treatments using medicinal plants have been used in traditional medicine. Andiroba is the name given by the Amazon people to the plant and oil from Carapa guianensis. The name "andiroba" comes from 
the Tupi-Guarani language and signifies the bitter taste of the oil. Carapa guianensis is a tree that grows throughout South America, West Indies and South Africa. In Brazil, this species is prevalently found in areas of the Amazon rainforest. Andiroba has been used as a medicine for fever, inflammation, and pain, as well as an anti-parasitic agent and insect repellent. The anti-inflammatory properties of andiroba are thought to depend on the presence of limonoids, and andiroba exhibits inhibitory effects on histamine, bradykinin, and platelet-activating factor actions ${ }^{4-6}$. Andiroba is registered in ANVISA (Brazilian National Health Surveillance Agency) as a hydrating cosmetic and insect repellent, but its clinical effectiveness makes it an adjuvant in the treatment of actinic dermatitis and compression bedsores. The wound healing activities of the ethanolic extracts of Carapa Guianensis have been demonstrated in rats $^{7}$, and improvement in the tensile strength of colonic open wounds in rats has been reported ${ }^{8}$. In humans, a prospective, comparative, double-blind study conducted in 33 patients showed that an andiroba-based emulsion applied to injuries caused by intense pulsed-light-based epilation was effective in preventing pain and erythema, effects similar to those seen with a low-potency corticoid ${ }^{9}$.

Therefore, the aim of this study was to evaluate the effects (on wound contraction, tensile strength, macrophages, myofibroblasts, and angiogenesis) of an emulsion based on andiroba oil on the healing of an excision fullthickness wound in Wistar rats.

\section{Methods}

This study was reviewed and approved by the Institutional Animal Care and Use Committee (Universidade Federal do Rio de Janeiro, protocol number 185/13). All Institutional Animal Care and Use Committee guidelines were followed throughout the entire experiment
Eighty-one male specific-pathogen-free Wistar rats, weighing approximately $250 \mathrm{~g}$, were obtained from the Center of Experimental Surgery, UFRJ, and were maintained in the animal facility of the Center. They were individually housed and allowed free access to a standard pellet diet and water ad libitum.

\section{Treatment}

A Brazilian commercial emulsion containing andiroba oil (Tegum ${ }^{\circ}$, Brazil) was topically applied to the incision wounds immediately after the surgery and every day until the end of the experiment.

\section{Experimental design}

Animals were randomly divided into two groups, consisting of wounded (Wound Group) and sham-operated animals (Sham Group). These groups were subdivided into treated (Wound + Treatment Group, and Sham + Treatment Group) and untreated (Wound Group, and Sham Group) groups. Each subgroup consisted of six animals.

\section{Anesthetic and surgical procedures}

Animals were anesthetized intraperitoneally (IP) with ketamine (Syntec, SP, Brazil $60 \mathrm{mg} / \mathrm{kg}$ ) and xylazine (Syntec, SP, Brazil $10 \mathrm{mg} / \mathrm{kg}$ ). The surgical interventions were carried out under sterile conditions. After shaving the dorsal region, a circular fullthickness skin excision wound $\left(500.0 \mathrm{~mm}^{2}\right)$ was created (Wound Group). Sham animals were shaved, and the dorsal region was manipulated for a period similar to the time required to perform the excision in the other group.

\section{Wound contraction ratio}

Macrophotographs of the wound/scar area were obtained at $3,7,15$, and 20 days postinjury. The wound areas $\left(\mathrm{mm}^{2}\right)$ were identified 
from macrophotography using image analysis software (Image-ProPlus version 4.5.1 [Media Cybernetics, Rockville, MD, USA]). Changes in the wound size are expressed as percentages of the contraction of the original wound (day 0 ).

Wound contraction ratio $(\%)=(A 0-A t) /$ $A 0 \times 100$

where $A 0$ and $A$ t are the initial wound area and the area at time $t$, respectively.

\section{Tissue harvesting}

Wounds or skin from the sham group were harvested on days 3, 7, 15 and 20 post-injury and were fixed in $10 \%$ buffered formaldehyde for 2 days. They were then submitted to a standard procedure for paraffin embedding.

\section{Histology}

Paraffin sections cut at a 5- $\mu \mathrm{m}$ thickness were stained with hematoxylin-eosin (HE) and modified picrosirius red (7- $\mu \mathrm{m}$ thick). Stained sections were blindly analyzed by a pathologist with a light microscope (Nikon Eclipse E-800, Japan). Picrosirius red-stained sections were analyzed using conventional optics or with a light microscope equipped with a filter to provide polarized illumination.

\section{Antibodies}

The following antibodies were used: anti- $\alpha$-smooth muscle actin, clone 1A4 (Dakocytomation, CA, USA, cat. M0851, monoclonal mouse) for detecting myofibroblasts/pericytes; anti-CD68 (AbdSerotec, NC, EUA, cat. MCA341R, monoclonal mouse) for macrophages; anti-Von Willebrand (factor VIII) (Dakocytomation, cat. A082, polyclonal rabbit) for endothelial cells; anti-iNOS (Thermo Scientific, USA, cat. RB-9242, polyclonal rabbit) for macrophage $\mathrm{M} 1$; antiarginase 1 (H-52) (Santa Cruz Biotechnologies, CA, USA, sc-20150, polyclonal rabbit; 1:100) for macrophage $\mathrm{M} 2$; anti-transforming growth factor $\beta 3$ (TGF $\beta 3$ ) (Santa Cruz Biotechnologies, sc-20150, polyclonal rabbit; 1:100), anti-rat collagen type III (Novotec, France, 20341, 1:100) and anti-rat type I (Novotec, 20141, 1:100).

\section{Immunohistochemistry}

Immunohistochemical procedures were performed on paraffin sections. Briefly, after dewaxing and rehydrating, sections were submitted to endogenous peroxidase inhibition $\left(3 \% \mathrm{H}_{2} \mathrm{O}_{2}\right.$ in methanol). A heatmediated antigen retrieval technique was performed in a microwave oven $(0.01 \mathrm{M}$ citrate buffer, pH 6.0, or Tris-EDTA buffer, $\mathrm{pH}$ 8.0) or with an enzyme for collagen staining $(0.2 \%$ hyaluronidase [from bovine testis, $\mathrm{H}-3506$, Sigma-Aldrich, St. Louis, USA]), according to the specific antibody. Then, after blocking the nonspecific binding of immunoglobulins to the tissue, primary antibodies were incubated for 16 hours at $4^{\circ} \mathrm{C}$ in a humidified chamber. The sections were then washed in $0.25 \%$ Tween phosphate-buffered saline (PBS) solution for 5 minutes, followed by secondary antibody incubation (Histofine Simple Stain Rat MAX PO (Mouse) and - PO (Rabbit) from Nichirei, Japan, cat. 414171F, and 414181F, respectively, both for rat tissue). The chromogen substrate was diaminobenzidine (Liquid DAB, Dako, cat. K3468). Negative control slides were incubated with mouse or rabbit isotype immunoglobulins or with the antibody diluent solution.

\section{Histomorphometry}

An image analysis system, consisting of a light microscope (Eclipse E800, Nikon, Japan) coupled with a digital camera (Evolution, Media Cybernetics Inc., Bethesda, MD, USA), and graphic interface software, Q-Capture 2.95.0 (version 2.0.5; Quantitative Imaging, Surrey, BC, Canada), were used. High-quality images 
(2048 × 1536-pixel buffer) were captured, and after program-setting calibration, images were analyzed using Image-Pro Plus software (version 4.5.1, Media Cybernetics, Rockville, MD, USA). The percentage of collagen-labeling (\%) was obtained from 15 or 8 images/animal, randomly obtained from histological sections using a $40 x$ objective lens.

\section{Number of $\mathrm{CD} 68^{+}$macrophages}

The number of macrophages was obtained from CD68-stained sections. The results are expressed as the number of macrophages/histological field \pm SEM.

\section{M1 or M2 macrophages}

The amount of M1 (iNOS reactive) or M2 (arginase-1 reactive) macrophages was obtained from iNOS- or arginase-1-stained slides. The results are expressed as M1 or M2 cell numbers/histological fields \pm SEM or the ratio $\mathrm{M} 1 / \mathrm{M} 2$.

\section{Number of myofibroblasts}

The number of myofibroblasts present in the wounds was obtained from $\alpha$-smastained slides. The results are expressed as the number of myofibroblasts/histological field \pm SEM.

\section{Angiogenesis}

Quantification of capillaries was performed by counting the number of capillaries (transversally cut) reactive to factor VIII-related antigen. The results are expressed as the number of capillaries/histological field \pm SEM.

Surface density of type I and type III collagens

The surface density of type- specific collagens deposited in the wound area on day 7 was assessed in 08 photomicrographs of col I- and col III-stained sections randomly obtained with a $x 40$ objective lens. The results are expressed as the percentage of collagen present in the wound area.

\section{TGF-83 quantification}

TGF 33 levels were quantified with a histological score obtained from 15 photomicrographs randomly obtained from the epidermis and dermis of TGF $\beta 3$-stained sections with a $\times 20$ objective lens. The histological score of epidermal TGF $\beta 3$ consisted of a numerical gradation where 0 : absence; 1 : TGF $\beta 3$ in the basal cells; 2 : TGF $\beta 3$ in the suprabasal layers; 3 : TGF $\beta 3$ in the basal and suprabasal layers; and 4: intense reactivity for TGF $\beta 3$ in all epidermal layers. For dermal TGF $\beta 3,0$ : absence of reactivity; 1 : TGF $\beta 3$ reactivity in inflammatory cells and fibroblasts; 2: TGF $\beta 3$ reactivity in endothelial and inflammatory cells/fibroblasts; 3: TGF- $\beta 3$ in vessel walls; and 4: TGF $\beta 3$ in the ECM. The sum of the epidermal and dermal numerical scores represents the numerical score for TGF $\beta 3$.

\section{Biomechanical test}

For the biomechanical assay (tensile breaking test), other groups of treated, nontreated, and sham animals ( $n=3$ /group) were prepared. Then, after 20 days post-surgery, a rectangular strip of skin containing the healed wound was harvested. The protocol used was that established by Garg and Paliwal ${ }^{10}$. A rectangular segment of skin containing the scar was cut perpendicularly to the long axis of the scar and was immediately bathed in chilled saline. Then, one end of the skin fragment was held by a gripping jaw and the other end was connected to a spring balance. Balance weights (weights of 1, 5, 10, 50 and $100 \mathrm{~g}$ ) were slowly and gradually added to the balance plate until scar rupture. The tensile breaking force is the 
weight required to rupture the scar, expressed in grams (g).

\section{Statistical analysis}

All numerical results are expressed as the mean \pm standard error of the mean (SEM). Data were analyzed using GraphPadPrism 5.0 (GraphPad Software, La Jolla, CA, USA). Statistical analysis was performed with the nonparametric t-test followed by the MannWhitney post hoc test or two-way analysis of variance followed by the Bonferroni or Tukey's post hoc test. Differences between groups were considered significant at $\mathrm{P}<0.05$.

\section{Results}

Treatment with the andiroba-based emulsion significantly enhanced the wound healing rates over time

Compared with the non-treated animals, topical use of the andiroba-based emulsion resulted in a significant enhancement of wound closure rates at all observed times $(P<0.05)$. Compared with the untreated wounds, the esthetic aspect was improved on D15 and D20 in the treated wounds (Table 1, Figure 1).

Table 1 - Wound healing effect of andiroba-based emulsion in an excision wound model.

\begin{tabular}{ccc}
\hline Parameter & Wound area $\left(\mathrm{mm}^{2}\right)$ and percentage of wound contraction \\
\hline Post-wounding days & Untreated Wounds & Treated Wounds \\
D0 & $549.0 \pm 1.7$ & $548.0 \pm 2.0$ \\
D3 & $501.0 \pm 0.29(8.74 \%)$ & $448.0 \pm 1.6(18.25 \%)$ \\
D7 & $407.9 \pm 0.29(25.50 \%)$ & $345.0 \pm 1.4(37.04 \%)$ \\
D15 & $355.0 \pm 1.9(35.33 \%)$ & $257.0 \pm 3.4(53.10 \%)$ \\
D20 & $268.0 \pm 1.5(51.18 \%)$ & $243.0 \pm 0.19(55.65 \%)$ \\
\hline
\end{tabular}

$\mathrm{N}=5$; values are expressed as the mean $\pm \mathrm{SEM}$

1

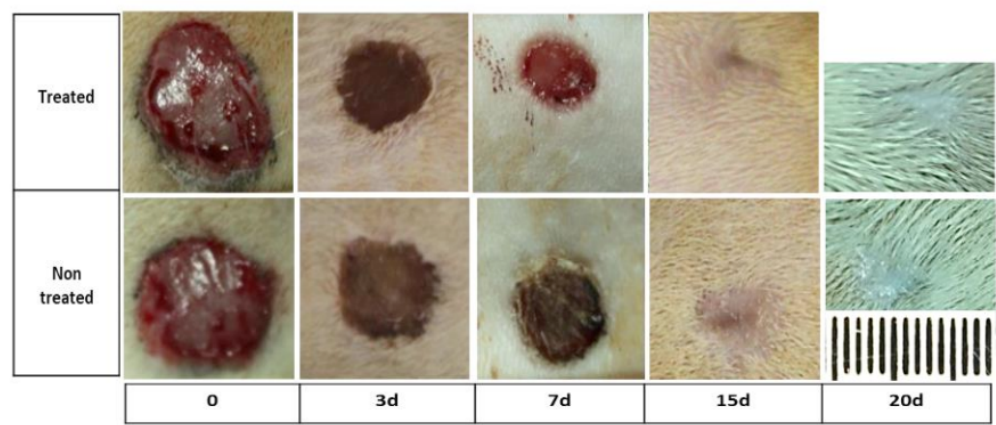

2

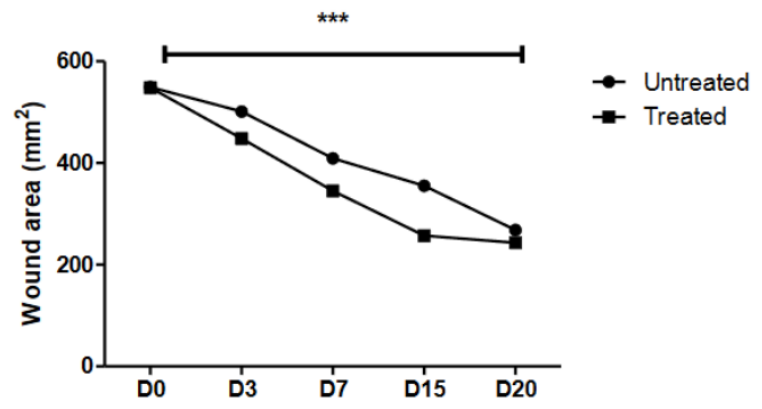

Figure 1 - Andiroba-based-emulsion-treated and non treated rat full-thickness skin wounds. A. Representative macrophotographs of andiroba-based emulsion- treated and untreated wounds over time; B. Graphical representation of treated and untreated wound area $\left(\mathrm{mm}^{2}\right)$ over time. Data are expressed as the mean \pm SEM and were submitted to two-way ANOVA followed by the Bonferroni post hoc test. ${ }^{* * * P}<0.001$. 
Treatment of wounds with the andiroba-based emulsion modified the histological pattern of wound healing over time

Compared with the untreated wounds, in the treated wounds on day 3 (D3), the level of inflammatory cells was apparently diminished in the wound (Figure 2A-B). On D7, the granulation tissue in both groups showed typical organization, where capillaries were interposed between fibroblasts and inflammatory cells (Figure 2C-D). In the untreated wounds, inflammation was still apparently evident (Figure 2C), but fibroblasts were disposed parallel to the dermal epidermal junction in the treated wounds (Figure 2D).
On D15, the treated and untreated wounds became re-epithelized, and a decrease in capillaries was apparent in both the untreated and treated groups (Figure 2E-F). The treated animals showed a more cellular wound at this stage (Figure 2F). On D20, the wounds were less cellular, and the ECM was composed of thick, collagen fibers in the untreated wounds, while the treated wounds showed a less dense collagen fiber network than the untreated wounds (Figure 2G-H). The histology of the SHAM groups was similar to that of normal skin but the SHAM-treated skin samples had a discreet hyperplastic epidermis (data not shown).

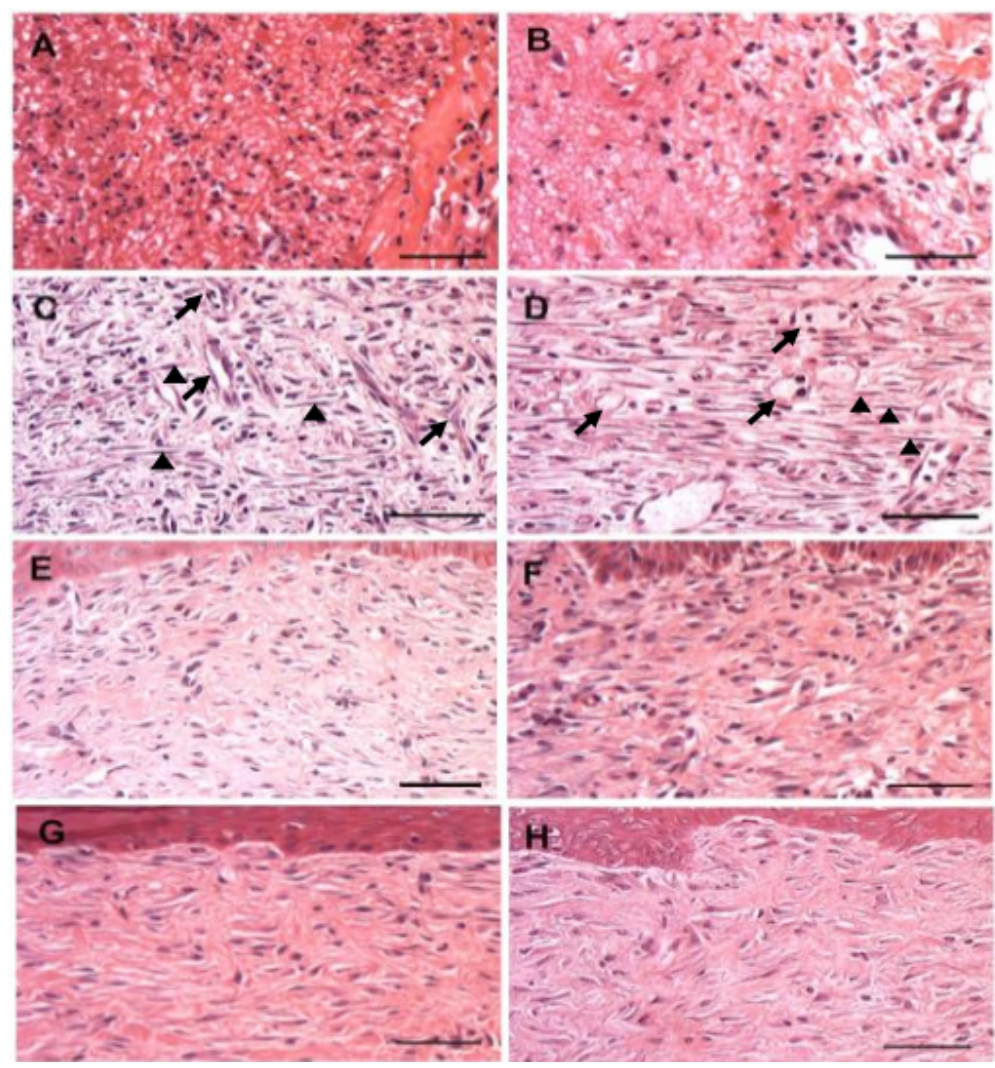

Figure 2 - Histological aspect of andiroba-based-emulsion-treated and untreated wounds over time. A. Untreated wound surface on D3 showing large quantities of inflammatory cells within the provisional matrix; B. Treated wound exhibiting few inflammatory cells; C. Untreated wound on D7. Fibroblasts (arrow head) mixed with inflammatory cells and new vessels (arrow); D. Treated wound on D7 showing a parallel orientation of fibroblasts (arrow head) among new vessels (arrow); E. Untreated wound on D15 showing deposition of collagen fibers according to hematoxylin-eosin staining. Scale bar: $100 \mu \mathrm{m}$. 
Compared with the untreated wounds, angiogenesis was more prominent in the treated wounds over time

Factor VIII immunostaining depicted the endothelial cells (Figure 3A-B) and enabled counting of the capillaries inside the wounds. The treated wounds showed more capillaries over time than did the untreated wounds (Figure 3A-C).

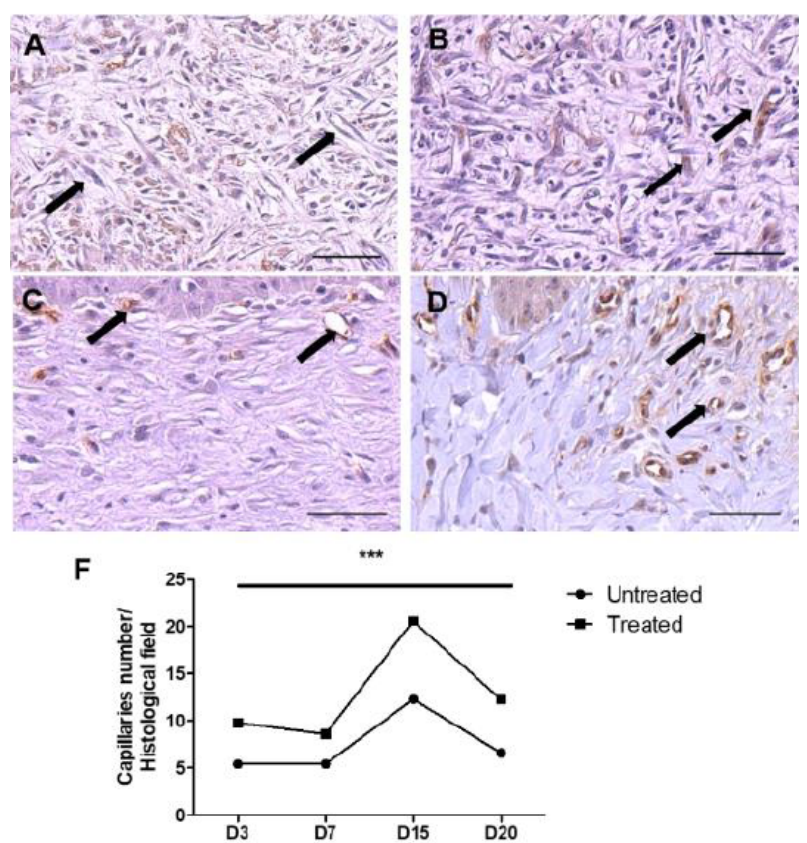

Figure 3 - Representative images and quantification of angiogenesis in andiroba-based-emulsion-treated and untreated wounds. Factor VIII-related antigen immunoenzymatic detection. Primary antibody (rabbit) revealed with anti-rabbit IgG conjugated to peroxidase. Diaminobenzidine used as the chromogen substrate for peroxidase. A. Untreated wound on D7. Note the capillaries lined with factor VIII-related antigen (arrow) among inflammatory cells; B. Treated wound on D7 exhibiting a large number of capillaries (arrow); C. Untreated wound on D15 containing few capillaries (arrow); D. Treated wound (D15) with a higher number of capillaries (arrow) compared with the untreated wounds. Scale bar: $100 \mu \mathrm{m}$; E. Images representing the angiogenesis quantification. Data represent the mean \pm SEM, and differences between groups were assessed by two-way ANOVA followed by the Bonferroni post hoc test. ${ }^{* * *} \mathrm{P}<0.001$.
Macrophages $C D 68^{+}$were present in the treated and untreated wounds, but their numbers were decreased in the treated animals

Compared with the untreated animals, the CD68+ macrophage population was significantly decreased over time in the wounds of treated animals, except on D20, when macrophages again increased in the treated rats (Figure 4A-E).

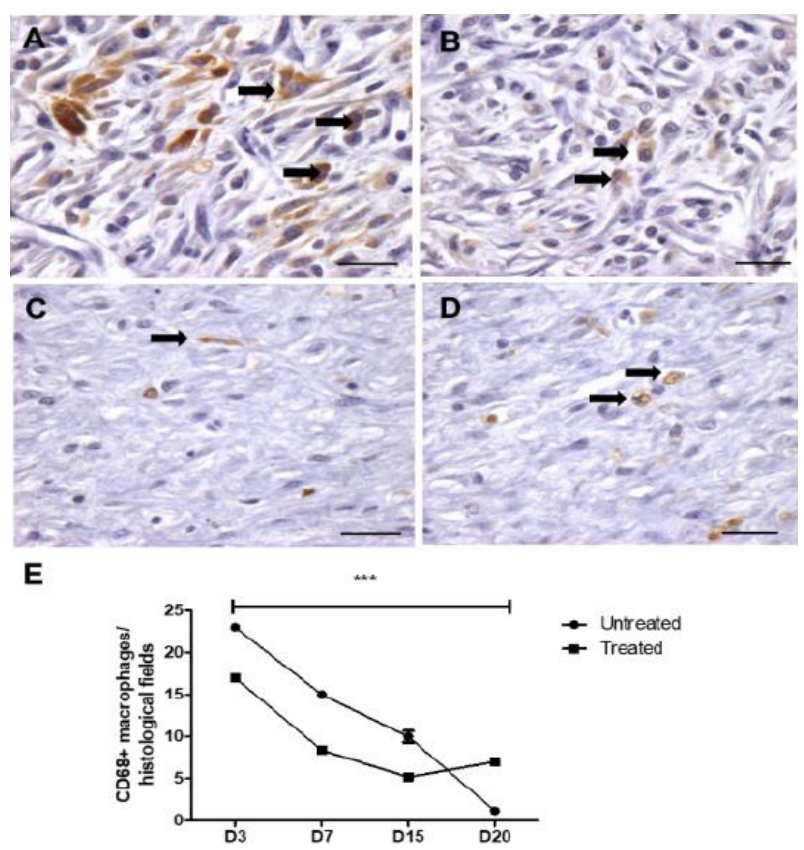

Figure 4 - Representative images of $\mathrm{CD}^{+} 8^{+}$ macrophages present in andiroba-based-emulsiontreated and untreated wounds and morphometry. A. Untreated wound on D7 showing a large number of $\mathrm{CD}^{+} 8^{+}$macrophages (arrow); B. Treated wound on D7 exhibiting few macrophages (arrow); C. Untreated wound on D15 containing few reactive macrophages (arrow); D. Treated wound (D15) with macrophages dispersed among the extracellular matrix (arrow). Scale bar: $50 \mu \mathrm{m}$; E. Graphical representation of macrophages during wound healing. Data represent the mean \pm SEM, and differences between groups were assessed by twoway ANOVA followed by the Bonferroni post hoc test. $* * * P<0.001$. 
M1 macrophages were present in both the treated and untreated wounds on day 3 postsurgery, but their numbers were higher in the treated animals

M1 macrophages (iNOS+) were also found in both groups on D3, but their numbers were higher in the treated animals (Figure 5AC). On other days, M1 macrophages were not found or were rarely found. On D3, the ratio $\mathrm{M} 1 / \mathrm{M} 2$ was more elevated in the treated wounds than in the untreated wounds (Figure $5 D)$.

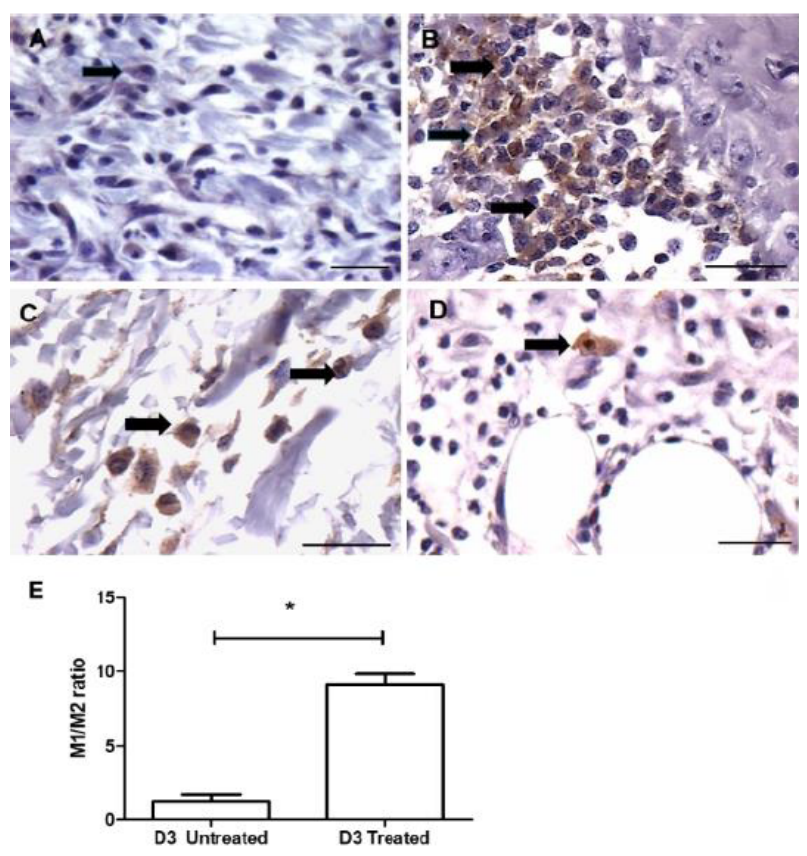

Figure 5 - Representative images of $\mathrm{M} 1$ (iNOS ${ }^{+}$) or $\mathrm{M}^{+}\left(\mathrm{Arg}^{+}\right)$macrophages present in andirobabased-emulsion-treated and untreated wounds and morphometry. A. Untreated wound (D3) showing rare iNOS $^{+}$macrophages (arrow); B. Treated wound on D3 showing foci of iNOS ${ }^{+}$macrophages inside the wound (arrow); C. Untreated wound on D3 containing some $\mathrm{Arg}^{+}$reactive macrophages (arrow); D. Treated wound (D3) with rare Arg1+ macrophages (arrow). Scale bar: $50 \mu \mathrm{m} ; \mathbf{E}$. Graphical representation of the M1/M2 ratio on D3. Data represent the mean \pm SEM, and differences between groups were assessed by non-parametric t-test followed by the Mann-Whitney post hoc test. $* \mathrm{P}<0.05$.

\section{M2 macrophages were present from $D 3$ to} D20 in the treated wounds

M2 macrophages (Arg1+) were present from D3 to D20 in both the untreated and the treated wounds. In the untreated wounds, the amount of M2 macrophages was higher than that in the treated animals from D3 to $\mathrm{D} 15$, when the number of $\mathrm{M} 2$ cells increased to greater levels than those found in the untreated animals (Figure 6A-E).

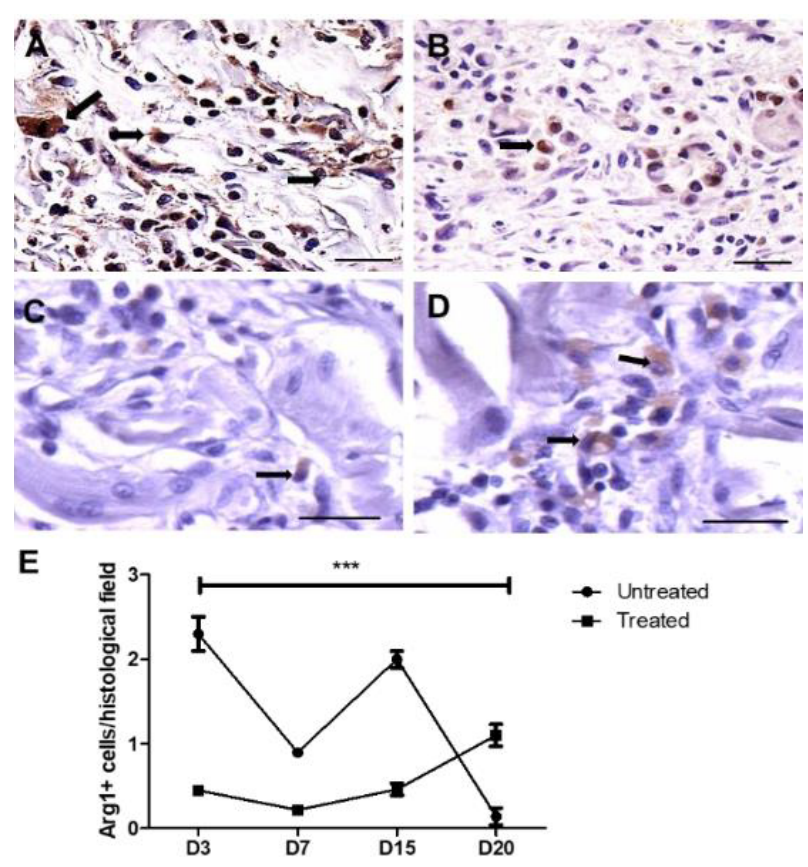

Figure 6 - Representative images of $\mathrm{M}^{+}\left(\mathrm{Arg} 1^{+}\right)$ macrophages present in andiroba-basedemulsion-treated and untreated wounds and morphometry. A. Untreated wound (D15) showing a large number of $\operatorname{Arg} 1^{+}$macrophages (arrow); B. Treated wound on D15 showing some Arg1 ${ }^{+}$macrophages inside the wound (arrow); C. Untreated wound on D20 containing rare $\operatorname{Arg} 1^{+}$ reactive macrophages (arrow); D. Treated wound (D15) with some Arg1+ macrophages (arrow). Scale bar: $50 \mu \mathrm{m}$; E. Graphical representation of M2 macrophage behavior during wound healing. Data represent the mean \pm SEM, and differences between groups were assessed by the two-way ANOVA non-parametric test followed by the Bonferroni post hoc test. ${ }^{* * *} \mathrm{P}<0.001$. 
Compared with the untreated wounds, the amount of myofibroblasts decreased in the andiroba-based-emulsion-treated wounds on D7 but persisted in the wounds on D20

In contrast to the treated wounds, myofibroblasts were seen on D7 in the untreated wounds, and cells were located in parallel to the dermal epidermal junction $(P<0.001)$ (Figure 7A-E). Compared with the untreated
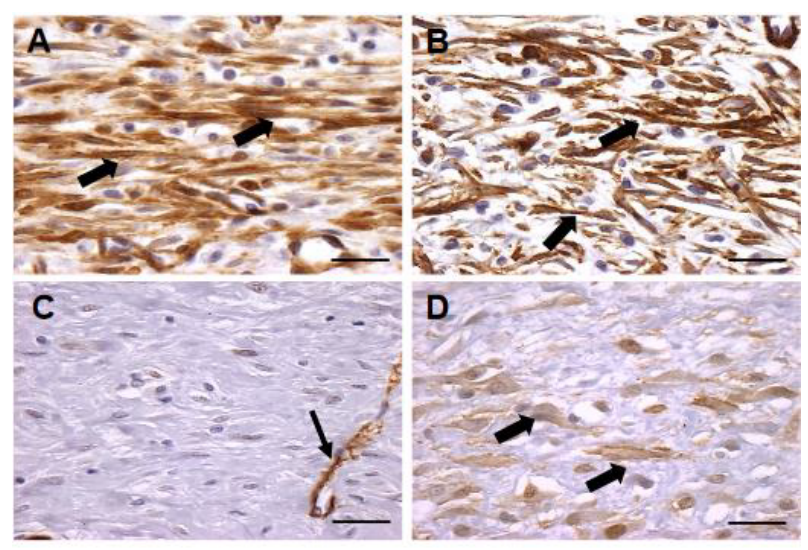

$\mathbf{F}$

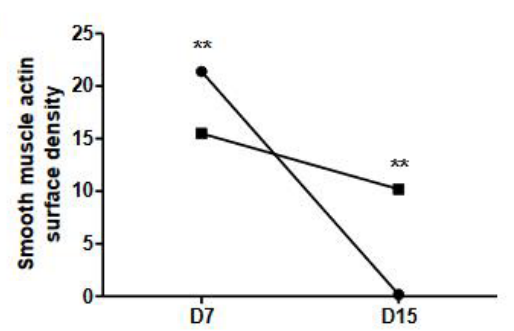

Figure 7 - Representative images of $\alpha$-sma ${ }^{+}$ myofibroblasts present in andiroba-basedemulsion-treated and untreated wounds and morphometry. A. Untreated wound (D7) showing a large number of $\alpha$-sma ${ }^{+}$myofibroblasts (arrow); B. Treated wound on D7 showing a lower number of $\alpha$-sma ${ }^{+}$myofibroblasts (arrow); C. Untreated wound on D15 negative for $\alpha$-sma ${ }^{+}$ myofibroblasts. Pericyte with $\alpha$-sma ${ }^{+}$reactivity in the blood vessel wall (arrow); D. Treated wound (D15) exhibiting some faintly stained $\alpha$-sma ${ }^{+}$ myofibroblasts (arrow). Scale bar: $50 \mu \mathrm{m}$; E. Graphical representation of $\alpha$-sma ${ }^{+}$myofibroblast behavior during wound healing. Data represent the mean \pm SEM, and differences between groups were assessed by the two-way ANOVA nonparametric test, followed by the Bonferroni post hoc test. ${ }^{* * P}<0.01$. wounds, in the treated wounds, the number of myofibroblasts was decreased on D7, and they were not present in an orderly direction (Figure 7B), similar to the cells in the untreated wounds (Figure 7A). On D15, myofibroblasts (Figure 7C) were still present in the treated wounds but were only faintly stained by $\alpha$-sma (Figure 7D). The figures show the significant differences in the level of myofibroblasts in both the treated and untreated wounds (Figure 7E).

\section{TGFB3 levels were increased over time in the treated wounds}

TGF 33 was absent in the skin of sham animals (Figure 8A). However, the skin from sham-treated animals exhibited TGF 33 in the basal layer of the epidermis (Figure $8 \mathrm{~B}$ ). On $D 3$, the untreated wounds showed TGF 33 in the regenerating keratinocytes at the borders of the wounds (Figure $8 \mathrm{C}$ ). However, on D3, TGFB3 was found in high levels in the treated wounds; this cytokine was present not only in the neoepidermis but also in the basal layer in the adjacent epidermis (Figure 8D).

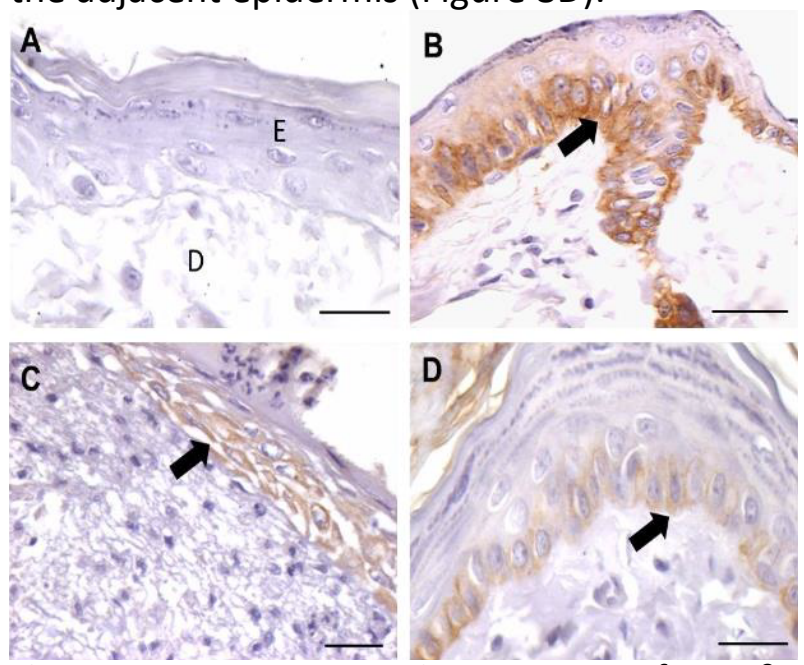

Figure $\mathbf{8}$ - Representative images of TGF $\beta 3$ immunohistochemistry. A. Skin obtained from a Sham animal (D3) showing absence of TGF 33 in the epidermis (E) or dermis (D); B. Skin from a Sham-treated animal (D3) showing reactivity for TGF 33 in the basal layer of a hyperplastic epidermis (arrow); C. Untreated wound on D3 exhibiting faint reactivity for TGF $\beta 3$ in migrating keratinocytes (arrow); D. Treated wound (D3) - hyperplastic epidermis adjacent to the wound faintly stained for TGFB3 (arrow). Scale bar: $50 \mu \mathrm{m}$. 
On D7, TGF $\beta 3$ reactivity was also seen in inflammatory cells in the dermis (Figure 9A) and in treated wounds (D7-T); TGF 33 was also present in some fibroblast-like cells (Figure $9 \mathrm{~B})$. On D15, all the regenerated and nonregenerated epidermises and inflammatory cells showed staining for TGF 33 , which was greater in the treated animals (Figure 9C). On D20, TGF 33 was present in the ECM, primarily in the treated wounds (Figure 9D). The graphical representation of the TGF $\beta 3$ histological score demonstrated that TGF $\beta 3$ levels were increased in both groups but were significantly increased after treatment (Figure 9E).

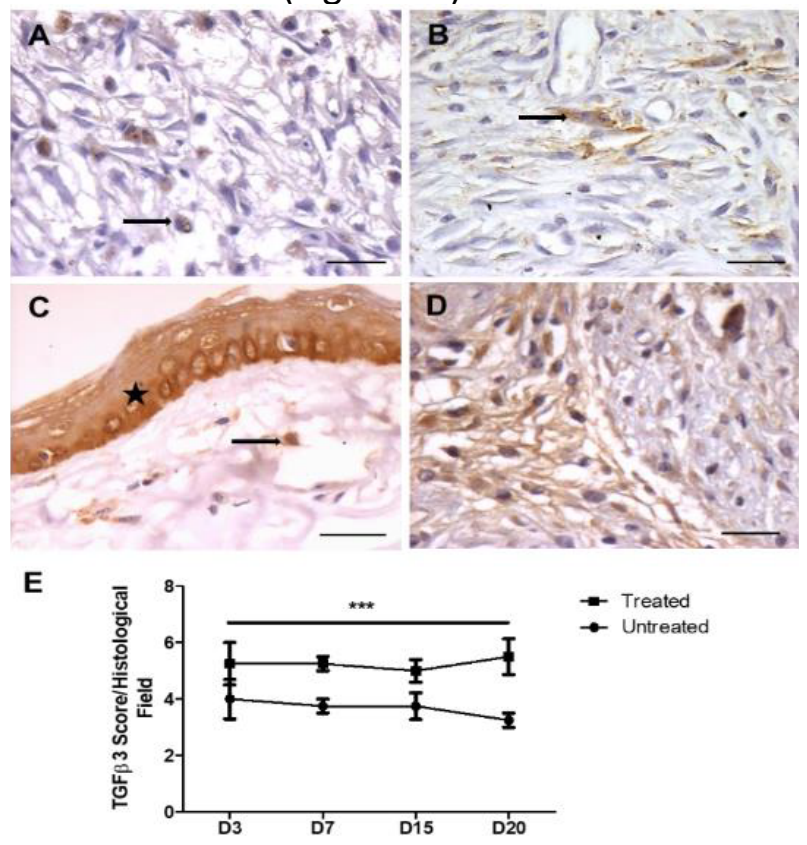

Figure 9 - Representative images of TGFB3 immunohistochemistry in andiroba-basedemulsion-treated and untreated wounds and morphometry. A. Untreated wound on D7 exhibiting some inflammatory cells in the ECM of the wound, which is faintly reactive for TGFB3 (arrow); B. Treated wound (D7) showing some fibroblast-like cells positive for TGF 33 (arrow); C. TGF 33 reactivity in all layers of the epidermis $\left({ }^{*}\right)$ adjacent to the treated wound (D15) and in an inflammatory cell in the dermis (arrow); D. Treated wound (D20) showing TGF 33 in the ECM of the wound $\left({ }^{*}\right)$. Scale bar: $50 \mu \mathrm{m}$; (E) Graphical representation of the TGF 33 histological score in treated and untreated wounds. Data represent the mean \pm SEM, and differences between groups were assessed by the two-way ANOVA non-parametric test, followed by the Bonferroni post hoc test. ${ }^{* * *} \mathrm{P}<0.005$.
On D7, the type I collagen level was similar between the untreated and treated wounds, but type III collagen was increased in the wounds treated with andiroba-based emulsion

Collagen type I was present in all dermises of both groups in a similar amount (Figure 10A-E). Type III collagen was significantly increased in the treated wounds (Figure 10C, $D$, and F).
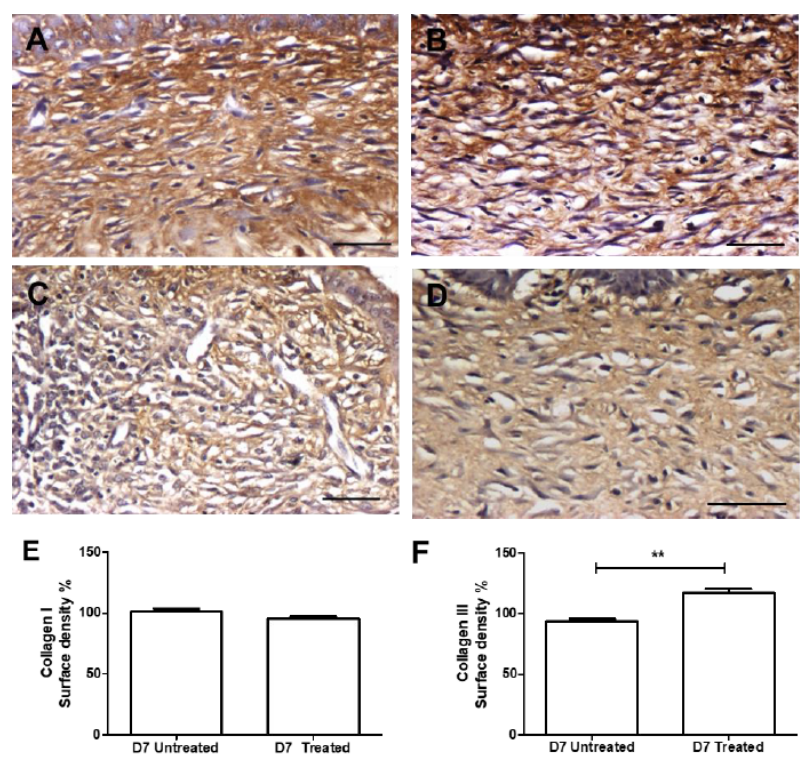

Figure 10 - Representative images of types I and III collagen immunohistochemistry on D7 in andirobabased-emulsion-treated and untreated wounds and morphometry. Collagen I immunostaining in an untreated wound (A) and in a treated wound (B); collagen III immunostaining in an untreated wound (C) and in a treated wound (D). Scale bar: 50 $\mu \mathrm{m}$; Graphical representation of collagen I (E) and collagen III (F). Data represent the mean \pm SEM, and differences between groups were assessed by the non-parametric t-test, followed by the Bonferroni post hoc test. ${ }^{*} \mathrm{P}<0.01$.

Polarized microscopy of picrosirius red revealed significant differences in collagen fibers in both the wounds treated with andiroba-based emulsion and the untreated wounds on D20

Picrosirius red polarized images of wounds revealed that the untreated wound 
collagen was predominantly composed of thick red fibers oriented parallel to each other (Figure 11A-B), while thin red or green fibers were oriented in a reticular pattern in the treated wounds (Figure 11C-D).

Tensile strengths of the andiroba-based emulsion treatment were increased up to $58.8 \%$ in the Sham Group on D20

As shown in Figure 10, on D20, the ultimate tensile strengths of the treated wounds were significantly higher than those in the untreated group $(P<0.001)$. In the treated wounds, the tensile strengths on D20 were $58.3 \%$ of the strengths in the sham group, while those of the untreated wounds were $45.8 \%$ of the strengths in the Sham Group (Figure 11E).
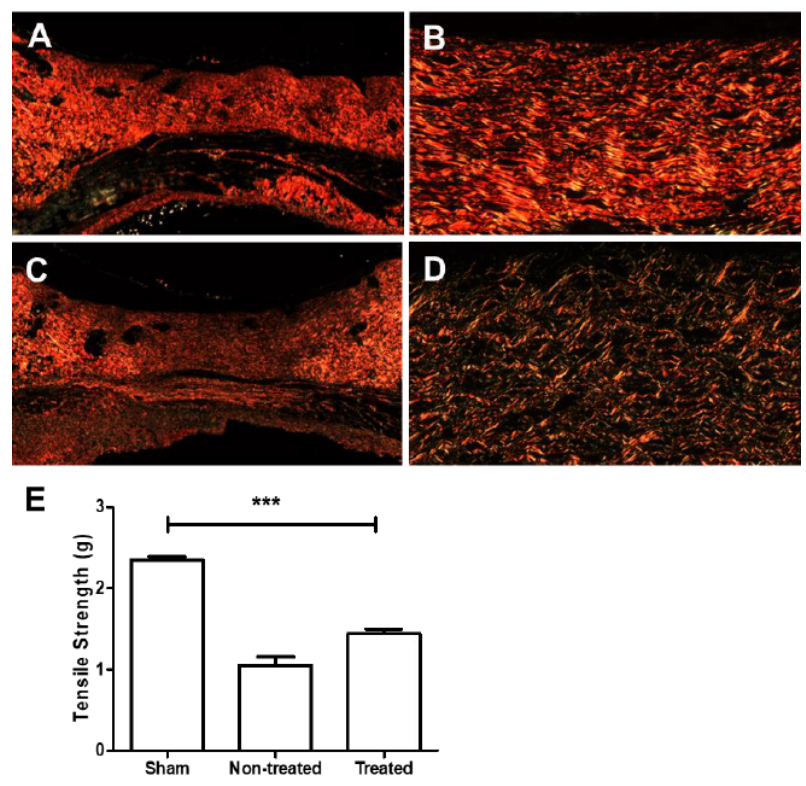

Figure 11 - Representative images of collagens stained with picrosirius red and viewed by polarization microscopy, as well as of biomechanical test results, in sham skin and andiroba-basedemulsion-treated or untreated wounds on D20. Low (A) and high (B) magnification of collagen in untreated wounds. Predominance of red, thick fibers (B) oriented parallel to the epidermal surface. Low (C) and high (D) magnification of collagen in a treated wound. (E) Tensile strength test results. Data represent the mean \pm SEM, and differences between groups were assessed by one-way ANOVA followed by Tukey's post hoc test. ${ }^{* *} \mathrm{p}<0.01$.

\section{- Discussion}

The results obtained herein show evidence that the andiroba-based emulsion is effective in improving the healing of fullthickness cutaneous wounds. Wound healing is a dynamic process with overlapping phases, which ultimately results in the healing of damaged tissues. Cutaneous wounds usually heal as a scar, which is weaker than intact skin and contain a disorganized ECM compared with that in unwounded skin and in early gestational fetal wounds ${ }^{1}$. These wounds differ from adult wounds in terms of the inflammatory response; the quality, quantity, and organization of the components of the ECM; the growth factors expressed; and the gene expression profile ${ }^{1}$.

Evidence suggests that the inflammatory response appears to be central to the evolution of wound healing. The activation of inflammatory cells provides the growth factors, cytokines, and chemokines necessary for the cross-talk between the dermis and epidermis, favoring the proliferation and migration of keratinocytes and the stimulation of mesenchymal cells ${ }^{11}$. Interestingly, fetal wounds heal without scar formation and are almost devoid of inflammatory cells due to the absence of a fully developed immune system. The absence of scarring is also seen in wounds induced in nude immunodeficient mice ${ }^{12}$. Indeed, these observations suggest a strong correlation between the immune system and regenerative capacity. Additionally, Urodele amphibians, such as newts, are capable of regenerating multiple organs, including skin and limbs. Compared with other amphibians, such as Xenopus, these amphibians are immunodeficient, which is associated with a more limited ability to regenerate organs. (Moreover, one of the alternatives to controlling scar formation after wounding is the control of inflammation ${ }^{14}$.

One such candidate is andiroba due to its striking anti-inflammatory property ${ }^{4}$. 
It seems that many of the constituents of andiroba oil, such as triterpenoids and limonoids ${ }^{15}$ have marked anti-inflammatory and anti-allergic properties. Some of the mechanisms involved in these properties are the impairment of signaling pathways triggered by histamine, bradykinin and platelet-activating factor as well as the inhibition of cytotoxicity induced by TNF- $\alpha$, LPS-induced NO production, and macrophage activation ${ }^{15}$. Moreover, these properties also block the activation of nuclear factor KB (NFKB) and, consequently, the production of inflammatory mediators responsible for myeloid cell recruitment, tumor necrosis factor- $\alpha$ (TNF- $\alpha$ ) and IL-1 $\beta$. Andiroba is also capable of inhibiting the activation of other cell populations, including mast cells, eosinophils and T lymphocytes ${ }^{6}$.

Herein, compared with untreated wounds, we showed that the topical use of andiroba-based emulsion during all the experiments was capable of modulating the inflammatory response, leading to a significant decrease in $\mathrm{CD}^{+} 8^{+}$macrophages up to D15. Apparently, the low level of inflammation seems to be beneficial in wound healing. The macrophage population plays key roles during wound healing, as it regulates repair and regeneration by modulation of the local inflammatory microenvironment, resolution of inflammation, and local sources of growth factors during various phases of wound healing ${ }^{16,17}$. Macrophages constitute a very versatile population with different states of activation, which depend on the microenvironment. They can be activated toward two opposite states, i.e., the M1 or classical and the $\mathrm{M} 2$ or alternative activation state $^{18}$. The M1 macrophages secrete various pro-inflammatory cytokines and nitric oxide $(\mathrm{NO})^{18}$. M2 macrophages have a high phagocytosis capacity and produce ECM components, angiogenic and chemotactic factors, TGF $\beta$, and IL-10 ${ }^{19}$, which are termed anti-inflammatory or pro-resolving due to their role in mitigating the inflammatory response ${ }^{18,19}$. Both phenotypes participate in wound healing. Early in wound healing, macrophages infiltrating the wound display a M1 phenotype $(85 \%)^{20}$, and upon phagocytosis of apoptotic cells, M1 macrophages die by apoptosis. The surviving M1 macrophages remain in the wound and transform into M2 macrophages, which contribute to the resolution of inflammation and the wound healing process ${ }^{21}$. In this study, we showed that the $M 1 / M 2$ ratio on D3 was higher in treated wounds than in untreated wounds. This finding possibly indicates that the treatment may have delayed the transformation of macrophages from $M 1$ to $M 2$, but the importance of this delay is not yet clear. However, some evidence has been obtained to help elucidate this finding. Evidence obtained from the depletion of specific macrophage populations during cutaneous wound healing showed that the depletion of an early pool of macrophages (D1-5) significantly delayed wound closure, but the macrophage repopulation of the wound rescued this delay with wound reepithelization by $\mathrm{D} 14$, similar to the results found in control animals $^{22}$. Furthermore, this depletion also yielded a significant reduction of the scar, suggesting a beneficial effect in terms of fibrosis. From D7 to D15, M2 macrophages had increased in both untreated and treated wounds, although the untreated levels remained higher than those in the treated wounds. The lowest level was observed in the treated group, suggesting better control of both inflammation and fibrogenesis in this group. However, on D20, the number of $M 2$ macrophages increased in the treated wounds. These changes may be related to modifications of the microenvironment, although regulation of these populations during wound healing is not clear. M2 macrophages suppress inflammatory responses and adaptive Th1 immunity and 
promote angiogenesis, tissue remodeling and repair $^{16}$. Additionally, M2 macrophages are a prominent source of TGF $\beta$ and are responsible for many aspects of wound repair, including inflammation, chemotaxis, wound contraction, angiogenesis and ECM deposition. This cytokine directly stimulates mesenchymal cells and fibroblasts to secrete collagen. TGF $\beta$ has 3 isoforms: 1, 2, and 3. TGF $\beta 1$ and TGF $\beta 2$ promote inflammatory cell recruitment and collagen production, while TGF $\beta 3$ is associated with reduced scarring through the promotion of collagen organization ${ }^{23}$. At gestational ages associated with scarless repair, TGF $\beta 1$ is expressed at low levels, while TGF $\beta 3$ is highly expressed, suggesting that the relative proportion of each subtype may be crucial for scarring. Additionally, the administration of exogenous TGF $\beta 3$ into a full-thickness wound for 3 days reduced the number of macrophages, increased neovascularization and decreased the levels of collagens I and III in the wound, resulting in markedly improved or absent scarring during adult wound healing ${ }^{23}$. Therefore, most of the effects seen in andirobatreated wounds seem to be related to the upregulation of TGF $\beta 3$ in the wounds.

Treatment with andiroba by the oral route, in another model of wound healing, also led to improvement of wound healing. However, these previous studies only describe an increase in the tensile strength of wounds, decreased inflammation ${ }^{8}$ or diminution of fibroblasts and increased angiogenesis. However, the results of this study demonstrated that the andiroba-based emulsion applied to the wound for up to 20 days was capable of modulating the presence of myofibroblasts and collagen levels. The decrease in myofibroblasts on D7 in the treated wounds may be explained by the possible reduction in TGF $\beta 1$ and/or TGF $\beta 3$ levels caused by the reduction in the number of macrophages, therefore impacting the differentiation of myofibroblasts. The myofibroblast population was increased on D15, while it would normally have disappeared. It is possible that the higher amount of type III collagen found in the ECM of treated wounds made it more compliant or less rigid, which altered the differentiation of the myofibroblasts, as already demonstrated in vitro. Fibroblasts cultured in compliant ECM or soft three-dimensional (3D) collagen gels show a less developed cytoskeleton with fewer stress fibers and $\alpha$-smooth muscle actin ${ }^{24}$. Indeed, myofibroblasts in early fetal wounds in vivo show markedly fewer $\alpha$-sma than did late fetal wounds or adult wounds ${ }^{25}$, similar to the finding in the present study. Fetal skin fibroblasts in culture also express less $\alpha$-sma, even if they are still able to construct collagen lattices ${ }^{26}$, but they are less efficient than those derived from adult skin ${ }^{27}$.

Moreover, although the treated wounds had a higher rate of wound contraction than the untreated wounds, elevated scars were not observed. In fact, it is known that fetal wounds may heal without contracture ${ }^{28}$ and that this ability is lost in late gestation.

The possible modified microenvironment of treated wounds, consequent to the topical treatment with andiroba-based emulsion, culminates in a greater synthesis of collagen III, which modifies the col III/collagen I ratio. For example, compared with the III/I ratio of 0.92 in untreated wounds, the ratio was 1.23 in the treated wounds on D7. The higher collagen III content in andiroba-treated wounds is similar to with that in the ECM of fetal wounds ${ }^{29}$, where, compared with the value of $10-20 \%$ seen in adult wounds, the collagen III network comprises $30-60 \%$ of the total collagen ${ }^{30}$.

\section{- Conclusion}

The topical application of andirobabased emulsion to wounds was capable of 
modulating levels of TGF $\beta 3$, macrophages, and myofibroblasts; angiogenesis; and collagen deposition, which in turn led to enhancement of the wound contraction rate, improvement in the tensile strength of the wounds and a more esthetic appearance.

\section{References}

1. Lo DD, Zimmermann AS, Nauta A, Longaker MT, Lorenz HP. Scarless fetal skin wound healing update. Birth Defects Res C Embryo Today. 2012;96:237-47. doi: 10.1002/ bdrc.21018.

2. Occleston NL, O'Kane S, Laverty HG, Cooper M, Fairlamb D, Mason T, Bush JA, Ferguson MW. Discovery and development of avotermin (recombinant human transforming growth factor beta 3): a new class of prophylactic therapeutic for the improvement of scarring. Wound Repair Regen. 2011;19 (Suppl. 1):S38-48. doi: 10.1111/j.1524-475X.2011.00711.x.

3. Singer AJ, Dagum AB. Current management of acute cutaneous wounds. N Engl J Med. 2008;359:1037-46. doi: 10.1056/ NEJMra0707253.

4. Burlando B, Cornara L. Revisiting Amazonian plants for skin care and disease. Cosmetics. 2017;4:25. doi: 10.3390/cosmetics4030025.

5. Penido C, Conte FP, Chagas MS, Rodrigues CA, Pereira JF, Henriques MG. Antiinflammatory effects of natural tetranortriterpenoids isolated from Carapa guianensis Aublet on zymosan-induced arthritis in mice. Inflamm Res. 2006;55:457-64. doi: 10.1007/s00011006-5161-8.

6. Henriques $M$, Penido $C$. The therapeutic properties of Carapa guianensis. Curr Pharm Des. 2014;20:850-6. doi: $10.2174 / 13816128113199990048$.

7. Nayak BS, Kanhai J, Milne DM, Swanston WH, Mayers S, Eversley M, Rao AV. Investigation of the wound healing activity of Carapa guianensis L. (Meliaceae) bark extract in rats using excision, incision, and dead space wound models. J Med Food. 2010;13:11416. doi: 10.1089/jmf.2009.0214.

8. Silva CE, Santos OJ, Ribas-Filho JM, Tabushi $\mathrm{FI}$, Kume MH, Jukonis LB, Cella IF. Effect of Carapa guianensis Aublet (Andiroba) and
Orbignya phalerata (Babassu) in colonic healing in rats. Rev Col Bras Cir. 2015;42:399406. doi: 10.1590/0100-69912015006009.

9 Cela EVSS, Rocha MB, Chia CY, Alves CF. Treatment of firstdegree burns with andiroba oil emulsion: a prospective, comparative, doubleblind study. Surg Cosmet Dermatol. 2014;6:44-9.

10.Garg V, Paliwal S. Wound-healing activity of ethanolic and aqueous extracts of Ficus benghalensis. J Adv Pharm Technol Res. 2011;2:110-4. doi: 10.4103/22314040.82957.

11.Pastar I, Stojadinovic O, Yin NC, Ramirez $\mathrm{H}$, Nusbaum AG, Sawaya A, Patel SB, Khalid L, Isseroff RR, Tomic-Canic M. Epithelialization in wound healing: a comprehensive review. Adv Wound Care (New Rochelle). 2014;3:44564. doi: 10.1089/wound.2013.0473.

12.Gawronska-Kozak B, Bogacki M, Rim J-S, Monroe WT, Manuel JA. Scarless skin repair in immunodeficient mice. Wound Repair Regen. 2006;14:265-76. doi: 10.1111/j.1743-6109.2006.00121.x.

13.Godwin JW, Rosenthal N. Scar-free wound healing and regeneration in amphibians: immunological influences on regenerative success. Differentiation. 2014;87:66-75. doi: 10.1016/j.diff.2014.02.002.

14. Rhett JM, Ghatnekar GS, Palatinus JA, O'Quinn M, Yost MJ, Gourdie RG. Novel therapies for scar reduction and regenerative healing of skin wounds. Trends Biotechnol. 2008;26:173-80. doi: 10.1016/j. tibtech.2007.12.007.

15. Ninomiya K, Miyazawa S, Ozeki K, Matsuo N, Muraoka O, Kikuchi T, Yamada T, Tanaka R, Morikawa T. Hepatoprotective limonoids from andiroba (Carapa guianensis). Int J Mol Sci. 2016;17:591. doi: 10.3390/ ijms17040591.

16.Delavary $B M$, van der Veer $W M$, van Egmond $M$, Niessen FB, Beelen RH. Macrophages in skin injury and repair. Immunobiology. 2011;216:753-62. doi: 10.1016/j.imbio.2011.01.001.

17.Wynn T, Barron L. Macrophages: master regulators of inflammation and fibrosis. Semin Liver Dis. 2010;30:245-57. doi: 10.1055/s-0030-1255354.

18.Sica A, Mantovani A. Macrophage plasticity and polarization: in vivo veritas. J Clin Invest. 2012;122:787-95. doi: 10.1172/JCI59643. 
19.Ferrante CJ, Leibovich SJ. Regulation of macrophage polarization and wound healing. Adv Wound Care. 2012;1:10-6. doi: 10.1089/wound.2011.0307.

20.Daley JM, Brancato SK, Thomay AA, Reichner JS, Albina JE. The phenotype of murine wound macrophages. J Leukoc Biol. 2010;87:59-67. PMID: 20052800.

21.Baum CL, Arpey CJ. Normal cutaneous wound healing: clinical correlation with cellular and molecular events. Dermatol Surg. 2005;31:674-86; discussion 86. PMID: 15996419.

22.Lucas T, Waisman A, Ranjan R, Roes J, Krieg T, Muller W, Roers A, Eming SA. Differential roles of macrophages in diverse phases of skin repair. J Immunol. 2010;184:3964-77. doi: 10.4049/jimmunol.0903356.

23.Shah M, Foreman DM, Ferguson MW. Neutralisation of TGF-beta 1 and TGF-beta 2 or exogenous addition of TGF-beta 3 to cutaneous rat wounds reduces scarring. J Cell Sci. 1995;108(Pt 3):985-1002. PMID: 7542672.

24.Tamariz E, Grinnell F. Modulation of fibroblast morphology and adhesion during collagen matrix remodeling. Mol Biol Cell. 2002;13:3915-29. doi: 10.1091/mbc.e0205-0291.

25.Estes JM, Berg JSV, Adzick NS, MacGillivray $T E$, Desmoulière A, Gabbiani G. Phenotypic and functional features of myofibroblasts in sheep fetal wounds. Differentiation. 1994;56:173-81. PMID: 8034132.

26. Moulin V, Auger FA, O'ConnorMcCourt $M$, Germain L. Fetal and postnatal sera differentially modulate human dermal fibroblast phenotypic and functional features in vitro. J Cell Physiol. 1997;171:1-10. doi: 10.1002/ (SICI)1097-4652(199704)171:1<1::AIDJCP1>3.0.CO;2-S.

27. Moulin V, Tam BY, Castilloux G, Auger FA, O'Connor-McCourt MD, Philip A, Germain L. Fetal and adult human skin fibroblasts display intrinsic differences in contractile capacity. J Cell Physiol. 2001;188:211-22. doi: 10.1002/jcp.1110.

28. Buchanan EP, Longaker MT, Lorenz HP. Fetal skin wound healing. Adv Clin Chem. 2009;48:137-61. PMID: 19803418.

29. Merkel JR, DiPaolo BR, Hallock GG, Rice DC. Type I and type III collagen content of healing wounds in fetal and adult rats. Exp Biol Med. 1988;187:493-7. PMID: 3353398.

30.Lovvorn HN, 3rd, Cheung DT, Nimni ME, Perelman N, Estes JM, Adzick NS. Relative distribution and crosslinking of collagen distinguish fetal from adult sheep wound repair. J Pediatr Surg. 1999;34:218-23. PMID: 10022176.

\section{Correspondence:}

Chang Yung Chia

Avenida das Américas, 505/ sala 203

22631-000 Rio de Janeiro - RJ Brasil

Tel.: (55 21)99649-5164

changplastica@gmail.com

Received: July 12, 2018

Review: Sept 09, 2018

Accepted: Oct 10, 2018
Conflict of interest: none

Financial sources: CNPq, CAPES, and FAPERJ 